\title{
A New Approach to Associating Human Impressions with Physical Features
}

\author{
Masahiro Sawai \\ Satellite Venture Business \\ Laboratory, Muroran Institute of \\ Technology \\ 27-1 Mizumoto-cho, Muroran \\ 050-8585, Japan
}

\author{
Yoshifumi Okada \\ College of Information and Systems, \\ Muroran Institute of Technology \\ 27-1 Mizumoto-cho, Muroran \\ 050-8585, Japan
}

\author{
Tomomasa Nagashima \\ College of Design and \\ Manufacturing, Muroran Institute of \\ Technology, \\ 27-1 Mizumoto-cho, Muroran \\ 050-8585, Japan
}

\begin{abstract}
A major aim of Kansei engineering is to translate human impressions into physical features of certain objects. When seeing a certain object, different individuals would try to express their impression of it with many different words even if they feel the same emotion for the object. However, no efficient approach to deal with such a large variety of impression words has been proposed so far. The aim of this study is to provide a new method for associating a large variety of impression words with physical features by introducing a new class of impression words called "meta-impressions." A meta-impression is defined as an impression whose relationship to physical features has been revealed in past studies. Focusing on the "music domain," we construct an M-I dictionary that describes the relationship between meta-impressions and various other impression words. The accuracy and applicability of our method are demonstrated experimentally.
\end{abstract}

\section{General Terms}

Text-mining, Information Retrieval, Natural Language Processing

\section{Keywords}

Kansei engineering, Impression, Physical feature, Thesaurus, Dictionary, Music

\section{INTRODUCTION}

Kansei engineering as proposed by Nagamachi has been attracting attention as a methodology for developing customeroriented products to meet customers' feelings and demands [1, 2]. "Kansei" is a Japanese word that means an individual's impressions/images evoked by external stimuli through the sensory organs. An important issue in Kansei engineering is correlating human impressions with physical features characterizing item such as music, pictures, clothes, and furniture.

Kansei engineering has recently been applied to impressionbased information systems for retrieving or recommending objects to match user's impressions [3-10]. In these systems, users must input a search query using several predefined impression words. Kumamoto et al. associated 20 impression words with particular physical features of music [3] and developed an impression-based music retrieval system [4]. However, such systems limit the variety of expressions that individuals can use because the selectable impression words are fixed. To successfully develop information systems based on individuals' Kansei, it is important to provide a flexible human interface to deal with a greater diversity of impression words.

To solve this problem, we consider a new class of impression words called "meta-impressions.." A meta-impression is an impression word that mediates relationships between impression words and particular physical features. They are introduced assuming that each human impression can be represented by a combination of meta-impressions. Here, a meta-impression is defined as an impression word whose relation to physical features has been experimentally or statistically revealed in past studies. Our aim is to provide a method for translating various impression words into physical features through these metaimpressions.

In this paper, we propose a new method that collects a large variety of impression words from electronic texts such as review comments and associates them automatically with metaimpressions. Then, an M-I dictionary is constructed to describe the relationship between meta-impressions and other impression words. In this study, an M-I dictionary specialized for the "music domain" is provided as an illustrative example. We show the usefulness of our method (and the M-I dictionary) by evaluating its adequacy and its application to an information retrieval system.

This paper is organized as follows. Section 2 describes the traditional Kansei engineering approach and its limitations. Section 3 explains the procedure for constructing the M-I dictionary. Section 4 shows the evaluation results of the M-I dictionary, and Section 5 applies the M-I dictionary to a music retrieval system. Finally, Section 6 summarizes our conclusions and suggests future work.

\section{TRADITIONAL APPROACH FOR KANSEI ENGINEERING AND ITS LIMITATION}

The typical procedure for associating impressions with physical features consists of four steps, as shown in Figure 1. The first step (Step1) collects as many impression words as possible in a certain domain of interest, such as music [3] or clothing design [2]. Next, redundant impression words with similar meanings are unified to a single representative word. In the second step (Step2), a questionnaire survey is performed to question experimental subjects about their impressions of objects with 


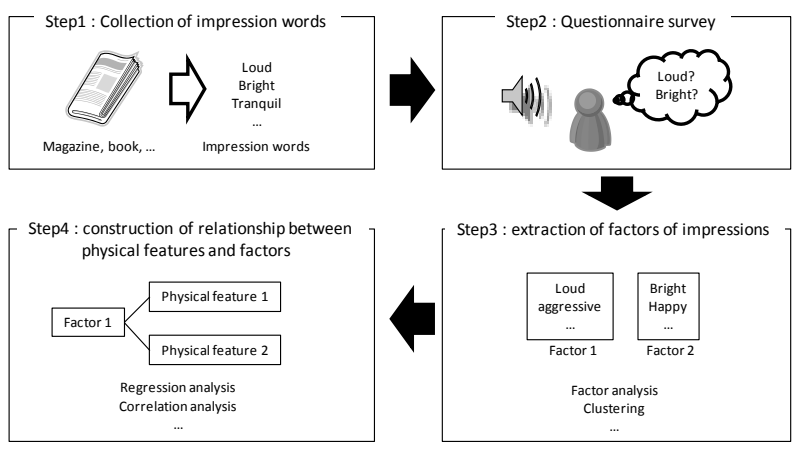

Figure 1. Typical procedure for investigating relationships between physical features and impressions in Kansei engineering

different physical characteristics (e.g., tempo in music), in which those objects are rated by multi-grade evaluation regarding each impression word obtained in Step.1. In the third step (Step3), multivariate analysis methods such as factor analysis and clustering are applied to group the impression words with similar trends (rating patterns) in the answers of the questionnaire survey. The final step (Step4) identifies physical features characterizing the factor of each group of impression words using regression analysis or correlation analysis.

Typically, Step1 requires much labor from the experimenters in collecting many impression words, and Step2 places a heavy burden on the subjects in rating them. In contrast, our method can automatically collect impression words from electronic texts using text mining. In addition, these impression words are associated with certain physical features through the metaimpressions; thus, laborious impression evaluation by subjects is not needed.

\section{METHOD}

Figure 2 shows an outline of our method; the shaded area represents the construction of an M-I dictionary, which is the focus of this study. In this section, we construct an M-I dictionary specialized for the "music domain" as an illustrative example. The M-I dictionary is obtained by the following three steps:

(1) Manually extract meta-impressions associated with certain physical features from the published literature.

(2) Collect synonyms of the meta-impressions as candidate impression words from a thesaurus.

(3) Identify unnecessary or redundant candidates for the music domain using text-mining and eliminate them by pruning.

\subsection{Extraction of Meta-impressions}

First, we extract meta-impressions, which are impression words whose relations to certain physical features have already been elucidated in the published literature. Kumamoto et al. associated 20 impression words with several physical features (tone color, pitch variation, volume variation, and so on) using multiple regression analysis [3, 4]. In this study, we employ these 20 impression words as meta-impressions.



Figure 2. Proposed method for associating various impression words with physical features through metaimmmocions

\subsection{Collection of Candidate Impression Words}

Second, we collect synonymous words (only adjectives and adjective verbs) for each meta-impression as candidate impression words by referring to a thesaurus. Those synonymous words are then linked to the corresponding meta-impressions.

\subsection{Pruning of Unnecessary Candidates}

In this step, a pruning process based on a text-mining approach is conducted to eliminate unnecessary candidate impression words that are generally not used in the music domain. First, we collect 46,178 review comments for a variety of audio CDs from amazon.co.jp [11]. Next, only adjectives and adjective verbs are extracted from these comments by a tagging tool $[12,13]$ that counts the appearance frequencies over all of the comments at the same time. By referring to the appearance frequencies, we prune candidate impression words that occur nine or fewer times. The M-I dictionary is provided as a set of links between the meta-impressions and the candidate impression words. In this dictionary, every impression word can be represented by a combination of multiple meta-impressions. Thus, an impression word can be translated into certain physical features through those meta-impressions.

\section{EVALUATION}

Using the above method, we obtain an M-I dictionary that includes a total of 399 impression words linked with all the meta-impressions. To evaluate the adequacy of the M-I dictionary, we conduct a questionnaire survey that require a yes or no answer about whether each link between an impression word and a meta-impression is adequate as a synonymous relation in the music domain. The subjects are eight male students. In this evaluation, 2 of the 20 meta-impressions are eliminated because no impression word is connected to them.

For each meta-impression, we count the number of "yes" answers for the connected impression words across the eight subjects. Figure 3 shows the percentage of "yes" answers for each metaimpression. We can see that more than half of the metaimpressions (10/18) present scores of more than $70 \%$; this shows 


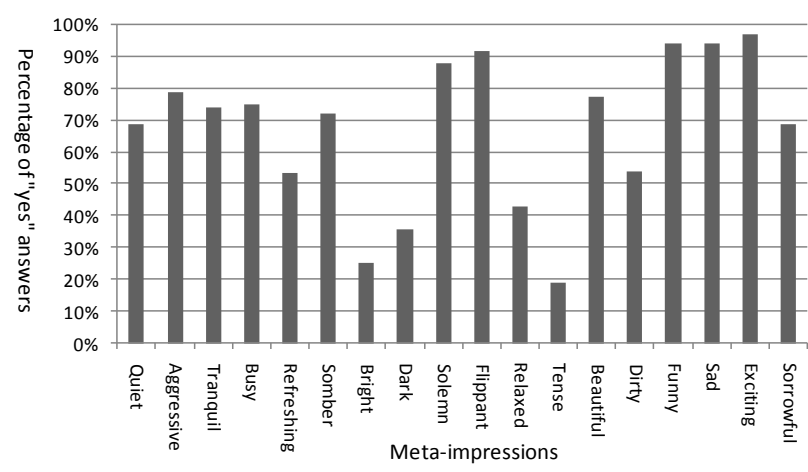

Figure 3. Results of the questionnaire survey

that the M-I dictionary characterizes the music domain well. In contrast, four meta-impressions ("bright," "dark," "relaxed," and "tense") show scores below 50\%, for the following reason. As seen in Table 1, the number of impression words related to these four meta-impressions is relatively large both before and after pruning. This means that these four meta-impressions link with many impression words unrelated to music. Since our method adopts a simple pruning operation based on the appearance frequency of impression words, such highly frequent but irrelevant impression words remain without being pruned. This problem is attributable to targeting only the "music domain" in the review comment processing. We will solve this problem by analyzing the differences in impression words between the music domain and various other domains.

\section{APPLICATION TO MUSIC RETRIEVAL SYSTEM}

The M-I dictionary is applied to a music retrieval system (the Impression-Based Music Retrieval System) developed by Kumamoto et al. [4] to demonstrate its practical usefulness.

The Impression-Based Music Retrieval System requires users' impressions of preferable music as the input. Impressions are specified using 10 pairs of adjectives (e.g., funny-sad). Note that the 10 pairs of adjectives (i.e., 20 adjectives) correspond to our 20 meta-impressions. Each pair of adjectives is placed at either end of a seven-point scale. By selecting one out of the seven values for each pair, users can obtain a list of music that is ranked on the basis of their rating values.

In this section, we convert user-specified impression words to meta-impressions using the M-I dictionary and then evaluate whether the retrieved music matches the user's impressions. First, we randomly select an impression word connected to each meta-impression and obtain 18 impression words in total. Second, these impression words are converted to the corresponding meta-impressions, and they are input to Kumamoto's system, in which the second values from the top/bottom are selected as the rating values because their descriptions accord exactly with the meta-impressions. Third, we present the top-ranked music to 12 male subjects and ask them to rate the match by a five-point scale in the same way as Kumamoto et al.: "agree (5)," "somewhat agree (4)," "neither
Table 1. Number of impression words before and after pruning

\begin{tabular}{|c|c|c|c|c|c|c|c|c|c|}
\hline & Quiet & Aggressive & Tranquil & Busy & Refreshing & Somber & Bright & Dark & Solem n \\
\hline Before prun ing & 101 & 63 & 37 & 24 & 52 & 42 & 243 & 224 & 26 \\
\hline \begin{tabular}{|l|} 
After pruning \\
\end{tabular} & 21 & 26 & 10 & 2 & 12 & 12 & 80 & 67 & 4 \\
\hline & Flippant & Rehxed & Tense & Beautiful & Dity & Funny & Sad & Exciting & Sorrow fill \\
\hline \begin{tabular}{|l} 
Before pruning \\
\end{tabular} & 17 & 89 & 139 & 99 & 176 & 3 & 44 & 11 & 32 \\
\hline After pruning & 3 & 28 & 43 & 28 & 30 & 2 & 17 & 4 & 10 \\
\hline
\end{tabular}

agree nor disagree (3)," "somewhat disagree (2)," and "disagree (1).."

As a result, the average score is 3.6, which is comparable to the score $(=3.8)$ Kumamoto et al. obtained directly by using metaimpressions. Their system restricts the usable impression words to 20, whereas the use of the M-I dictionary enables the use of a wider variety of impression words. Thus, we expect that the M-I dictionary can enhance the flexibility of media retrieval and recommendation based on users' impressions.

\section{CONCLUSIONS}

We proposed a method for automatically constructing an M-I dictionary that associates a large variety of impression words with fewer meta-impressions by text-mining. In this study, we made an M-I dictionary specialized for the "music domain" and showed its adequacy and applicability for practical use. We believe that our method a promising approach for not only dealing with many different impressions having the same meaning but also reducing laborious and time-consuming tasks such as collecting impression words and responding to questionnaire surveys.

In the future, we will improve the accuracy of the pruning process. We will also apply our method to different domains in addition to the music domain and develop retrieval and recommendation systems implementing the M-I dictionaries.

\section{REFERENCES}

[1] Nagamachi, M. 1995. "Kansei engineering as a powerful consumer-oriented technology for product development", International Journal of Industrial Ergonomics, Vol. 15, 311.

[2] Nagamachi, M. 1989. "Kansei Engineering", Kaibundo Publishing, Japan, in Japanese.

[3] Kumamoto, T., and Ohta, K. 2002. "Automatically and Numerically Expressing Impressions of a Music Piece for Music-Retrieval based on User's Impressions", IPSJ SIG Notes, Vol. 2002, No. 63, 37-42, in Japanese.

[4] Kumamoto, T., and Ohta, K. "Implementation and Evaluation of our System of Retrieving Music Pieces with Similar Impressions by Inputting the Impressions of a Target Music Piece", IPSJ SIG Notes, Vol. 2002, No. 41, 89-96, 2002, in Japanese.

[5] Akira, S., Jun, O and Hajime, K. 2000. "An Impressionbased Retrieval System of Music Collection", Proc. of 4th 
Int. Conf. on Knowledge-Based Intelligent System and Allied Technologies, Brighton, U.K., 856-859.

[6] Takayama, T., Sasaki, H. and Kuroda, S. 2005. "Personalization by Relevance Ranking Feedback in Impression-based Retrieval for Multimedia Database", Journal of Systemics, Cybernetics and Informatics, Vol.3, No.2, 85-89.

[7] Kikuchi, Y., Hashimoto, T., Ikeda, T. and Murata, Y. 2006. "Extension and Evaluation of Interface "2D-RIB" for Impression Based Retrieval", World Academy of Science, Engineering and Technology 15, 37-42.

[8] Kuroda, K. and Hagiwara, M. 2002. "An image retrieval system by impression words and specific object namesIRIS", Neurocomputing, Vol.43, No.1-4, 259-276
[9] Hayashi, T. and Hagiwara, M. 1998. "Image query by impression words the IQI system", IEEE Trans. Consumer Electron, Vol.44, No.2, 347-352.

[10] Kurita, T. and Kato, T. 1993. "Learning of Personal Visual Impression for Image Database Systems", Proc. of the Second Int. Conf. on Document Analysis and Recognition, $547-552$

[11] Amazon.co.jp, [Online], Available: http://www.amazon.co.jp/

[12] Matsumoto, Y. 2000. "Morphological Analysis System Chasen $(<$ Special Features $>$ Easy to Use Practical Freeware for Natural Language Processing) ", Journal of IPSJ, Vol. 41, No. 11, 1208-1214, in Japanese.

[13] Morphological Analysis System Chasen. [Online], Available: http://chasen-legacy.sourceforge.jp/ 